\title{
L'agonista del recettore GLP-1 promuove il rimodellamento del tessuto adiposo bruno nel tessuto adiposo bianco murino tramite SIRT1
}

\author{
Andrea Fabbri ${ }^{1}$
}

Pubblicato online: 30 novembre 2016

(c) Springer International Publishing AG 2016

\section{Commento a:}

GLP-1 receptor agonist promotes brown remodelling in mouse white adipose tissue through SIRT1.

X. Fen, L. Beisi, Z. Xiaobin, C. Zonglan, C. Huaniy,

X. Haixia, L. Hua, W. Jianping.

Diabetologia (2016) 59(5):1059-1069

Gli agonisti del recettore GLP-1 (GLP-1R), sostanze ipoglicemizzanti note per la loro attività insulinotropa glucosiodipendente, hanno un marcato effetto di riduzione di peso nel diabete tipo 2 e nell'obesità. I meccanismi noti per questa azione sono l'inibizione dell'appetito e dell'assunzione di cibo tramite un'azione diretta a livello ipotalamico e del sistema parasimpatico. Tuttavia, poco si conosce circa l'attività di GLP-1R nel tessuto adiposo.

Studi recenti hanno dimostrato che il browning del tessuto adiposo, incluso il rimodellamento bruno del tessuto adiposo bianco (WAT) e lo stimolo della funzione del tessuto adiposo bruno (BAT), potrebbe contrastare l'obesità. Il rimodellamento bruno del WAT conferisce aspetti simil-BAT al WAT e lo rimodella conferendogli una capacità di dissipazione di energia piuttosto che solo una capacità di sito di accumulo di energia. Sirtuina 1 (SIRT1), una deacetilasi $\mathrm{NAD}^{+}$-dipendente coinvolta nel metabolismo lipidico, è stata proposta come target terapeutico potenziale per trattare i disturbi metabolici correlati con l'obesità. L'aumento di attività di SIRT1 determina l'attivazione della triacilglicerol-lipasi (ATGL) adipocitaria, con conseguente deplezione di triacilglicerolo di WAT. Inoltre, l'overespressione di SIRT1 nel WAT determina una riduzione dell'ac-

A. Fabbri

andrea.fabbri@uniroma2.it

1 UOC di Endocrinologia, Ospedale CTO_ASL Roma 2, Dipartimento di Medicina Interna, Università degli Studi di Roma Tor Vergata, Roma, Italia cumulo di grasso negli adipociti e un aumento della spesa energetica dell'intero organismo.

Date queste premesse, in questo studio gli autori hanno valutato il possibile contributo del rimodellamento bruno di WAT nell'effetto di riduzione di peso determinata dall'agonista del GLP-1R exenatide e il ruolo di SIRT1 in questo processo in un modello murino, tramite studi in vivo e in vitro. Negli studi in vivo sono stati utilizzati topi di controllo (C5TBL/6J) e topi mutati SIRT1 ${ }^{+/-}$trattati con il GLP-1R agonista exenatide $(24 \mathrm{nmol} / \mathrm{kg})$ per 8 settimane in presenza di dieta ad alto contenuto di grassi (HFD) dopo 12 settimane di dieta HFD. Negli studi in vitro sono stati utilizzati adipociti differenziati 3T3L1 resi knock-out per il gene SIRT1. I risultati hanno dimostrato che: (1) in vivo l'exenatide è stata in grado di ridurre la massa grassa e di aumentare la capacità lipolitica e ossidativa di WAT attraverso la biogenesi mitocondriale nei topi di controllo resi obesi dalla HFD e questi effetti erano marcatamente minori nei topi $S I R T 1^{+/-}$; e (2) in vitro l'agonista GLP-1R exendina-4 è stato in grado di aumentare la lipolisi e l'ossidazione degli acidi grassi tramite l'aumento dell'espressione e attività di SIRT1 negli adipociti differenziati 3T3L1 e questo effetto veniva attenuato nelle cellule 3T3L1 rese knock-out per SIRT1.

Le conclusioni di questo studio molto elegante e innovativo dimostrano che lo stimolo del recettore GLP-1R promuove il rimodellamento bruno del tessuto adiposo bianco in maniera SIRT1-dipendente e che questo potrebbe essere uno dei meccanismi di tipo periferico responsabili della sua potente azione dimagrante. Inoltre, questi risultati rivelano nuovi target terapeutici per il trattamento dell'obesità e dei disordini metabolici ad essa associati. 